\title{
ARTICLE
}

\section{Third-order magnetic-susceptibility of itinerant-electron metamagnet UCoAl}

\author{
Mizuho Maeda $^{\mathrm{a}^{*}}$, Takuya Asai ${ }^{\mathrm{a}}$, Takemi Komatsubara ${ }^{\mathrm{b}}$, Tomoo Yamamura ${ }^{\mathrm{b}}$ and Noriaki Kimura ${ }^{\mathrm{a}, \mathrm{c}}$ \\ ${ }^{a}$ Department of Physics, Tohoku University, Sendai 980-8578, Japan; ${ }^{b}$ Institute for Materials Research, Tohoku University, Sendai \\ 980-8577, Japan; ${ }^{C}$ Center for Low Temperature Science, Tohoku University, Sendai 980-8578, Japan
}

\begin{abstract}
We report the third harmonics of the AC susceptibility in the itinerant-electron metamagnetic compound UCoAl. The third harmonics $\chi_{3}^{\prime}$ shows a sharp peak near the critical point of the metamagnetic transition line although a critical divergence toward the critical point is absent. The critical point can be determined as the onset of the peak in $\chi_{3}^{\prime}$, which is sharper than the peak in the fundamental component of the AC susceptibility, $\chi_{1}^{\prime}$. This result suggests that the measurement of $\chi_{3}^{\prime}$ can be a new tool for the determination of the quantum critical point in itinerant-electron metamagnetic system.
\end{abstract}

Keywords: itinerant-electron metamagnetism; AC susceptibility; nonlinear susceptibility; critical point; $\mathrm{UCo} A \boldsymbol{l}$

\section{Introduction}

Quantum criticality is one of the most interesting phenomena in solid state physics. For some itinerant-electron magnetic compounds, anomalous phenomena such as non-Fermi liquid, unconventional superconductivity, and spin nematic phase are found near the quantum critical point (QCP) $[1,2]$. Among them, uranium compounds provide a rich field of investigation for the understanding of the quantum criticality $[3,4]$.

$\mathrm{UCoAl}$ is an itinerant-electron magnetic compound, whose quantum critical point (QCP) can be reached by applying magnetic field and pressure. It crystallizes in the ZrNiAl-type $P \overline{6} 2 m$ hexagonal structure [5]. The ground state of UCoAl is paramagnetic and exhibits an itinerant-electron metamagnetic transition at $0.6 \mathrm{~T}$. The transition line in the $H-T$ plane ends at the critical point $(\mathrm{CP})\left(T_{\mathrm{cr}}, H_{\mathrm{cr}}\right)$ and the transition changes to a crosssover above $T_{\mathrm{cr}}$. Some experiments have been reported on this compound to reveal the quantum critical phenomena of the itinerant-electron ferromagnetic system. The transverse magnetoresistance measurement showed that the $A$ coefficient in the resistivity is enhanced at $\approx 1.5 \mathrm{GPa}[6]$. A divergence of the $A$ coefficient is characteristic of the magnetic QCP. On the other hand, the AC susceptibility measurement showed that the magnetic hysteresis vanishes at $2.9 \mathrm{GPa}$, which indicates that the first-order metamagnetic transition persists at $1.5 \mathrm{GPa}$ and that the QCP is located at 2.9 $\mathrm{GPa}$ [7]. However, the critical divergence in the linear susceptibility was not significant at $2.9 \mathrm{GPa}$. A more

*Corresponding author.Email: mmaeda@mail.clts.tohoku.ac.jp exact determination of the location of the QCP is thus necessary for the understanding of the quantum critical phenomena of this system.

In general, higher-order magnetic susceptibility $\chi_{n}=\frac{1}{n !} \frac{\mathrm{d}^{n} M}{\mathrm{~d} H^{n}},(n>1)$ diverges stronger than the linear susceptibility $\chi_{1}$ at the magnetic critical point [8-10]. Indeed, a critical divergence with a larger critical index of $\chi_{3}$ toward the Curie temperature $T_{\mathrm{C}}$ is experimentally verified in several ferromagnets $[11,12]$. This motivated us to utilize higher-order susceptibility for the determination of the metamagnetic CP and QCP. In this report, we show the first trial of the measurement of the third harmonics in the AC susceptibility on $\mathrm{UCoAl}$ at ambient pressure.

\section{Experimentals}

Single crystals of UCoAl were grown by the Czochralski pulling method in a tetra-arc furnace. The dimension of the sample (\#1) was 1.9 (a-axis) $\times 3.9$ $\left(a^{*}\right.$-axis $) \times 0.31(c$-axis $) \mathrm{mm}^{3}$. The residual resistivity and its ratio were $16.4 \mu \Omega \mathrm{cm}$ and $\approx 10$, respectively, where the electrical current was applied along the hexagonal $a^{*}$-axis. Another sample (\#2) with different dimension, 0.75 (a-axis) $\times 0.75$ ( $a^{*}$-axis $) \times 4.35$ (c-axis) $\mathrm{mm}^{3}$ was also prepared to check the effect of demagnetization.

Higher-order susceptibilities $\chi_{n}$ appears as the coefficient of the Taylor expansion of magnetization $M(H)$ around $H=H_{0}$ :

$$
\begin{gathered}
M(H)=M\left(H_{0}\right)+\chi_{1}\left(H-H_{0}\right)+\chi_{2}\left(H-H_{0}\right)^{2} \\
+\chi_{3}\left(H-H_{0}\right)^{3}+\chi_{4}\left(H-H_{0}\right)^{4}+\cdots
\end{gathered}
$$


As we measured AC susceptibility, the magnetic field $H$ can be written as the sum of the static field $H_{0}$ and the excitation field $h_{0} \cos \omega t$, namely, $H=H_{0}+$ $h_{0} \cos \omega t$. Here, $\omega / 2 \pi=f$ and $h_{0}$ are the frequency and amplitude of the excitation field, respectively. Hence we obtain from Eq. (1),

$$
\begin{aligned}
M(t) & =\left(M\left(H_{0}\right)+\frac{1}{2} \chi_{2} h_{0}^{2}+\frac{3}{8} \chi_{4} h_{0}^{4}+\cdots\right) \\
& +\left(\chi_{1}+\frac{3}{4} \chi_{3} h_{0}^{2}+\frac{5}{8} \chi_{5} h_{0}^{4}+\cdots\right) h_{0} \cos \omega t \\
& +\left(\frac{1}{2} \chi_{2}+\frac{1}{2} \chi_{4} h_{0}^{2}+\cdots\right) h_{0}^{2} \cos 2 \omega t \\
& +\left(\frac{1}{4} \chi_{3}+\frac{5}{16} \chi_{5} h_{0}^{2}+\cdots\right) h_{0}^{3} \cos 3 \omega t
\end{aligned}
$$

Here, we ignored the effect of phase delay for simplicity. The AC susceptibility was measured by a mutual inductance method. The voltage induced in a pick-up coil $V_{\text {sig }}$ is proportional to the change in $M(t)$ :

$$
\begin{aligned}
V_{\text {sig }} & \propto-\frac{\mathrm{d}}{\mathrm{d} t} M(t) \\
& \propto\left(\chi_{1}+\frac{3}{4} \chi_{3} h_{0}^{2}+\frac{5}{8} \chi_{5} h_{0}^{4}+\cdots\right) \omega h_{0} \sin \omega t \\
& +\left(\frac{1}{2} \chi_{2}+\frac{1}{2} \chi_{4} h_{0}^{2}+\cdots\right) 2 \omega h_{0}^{2} \sin 2 \omega t \\
& +\left(\frac{1}{4} \chi_{3}+\frac{5}{16} \chi_{5} h_{0}^{2}+\cdots\right) 3 \omega h_{0}^{3} \sin 3 \omega t
\end{aligned}
$$

Therefore, the real part of the $n$-th harmonics in the AC susceptibility, $\chi_{\mathrm{n}}^{\prime}$, becomes

$$
\begin{aligned}
& \chi_{1}^{\prime} \sim \omega h_{0} \chi_{1}, \\
& \chi_{3}^{\prime} \sim \omega h_{0}^{3} \chi_{3} .
\end{aligned}
$$

Here we ignored the higher-order terms in each parenthesis of Eq. (3), assuming that $h_{0}$ is small enough.

We used two lock-in amplifiers (LIA) (SR830, Stanford Research Systems, Inc.) to detect simultaneously the fundamental $(n=1)$ and the third $(n=3)$ harmonics in $V_{\text {sig. }}$ For the latter LIA, the phase shift of the lock-in reference signal was set to three times larger than that of the former LIA. The frequency $f$ and amplitude $h_{0}$ of the excitation field were typically $67 \mathrm{~Hz}$ and $0.98 \mathrm{Oe}$, respectively. The data shown below was normalized by $f h_{0}$ for $\chi_{1}^{\prime}$ and $f h_{0}^{3}$ for $\chi_{3}^{\prime}$ to omit both $f$ and $h_{0}$ dependences. The magnetic field was applied along the $c$-axis. DC magnetization was measured using a Magnetic Property Measurement System (Quantum Design, Inc.).

\section{Results and discussion}

\subsection{Measurement of $\chi_{3}^{\prime}$}

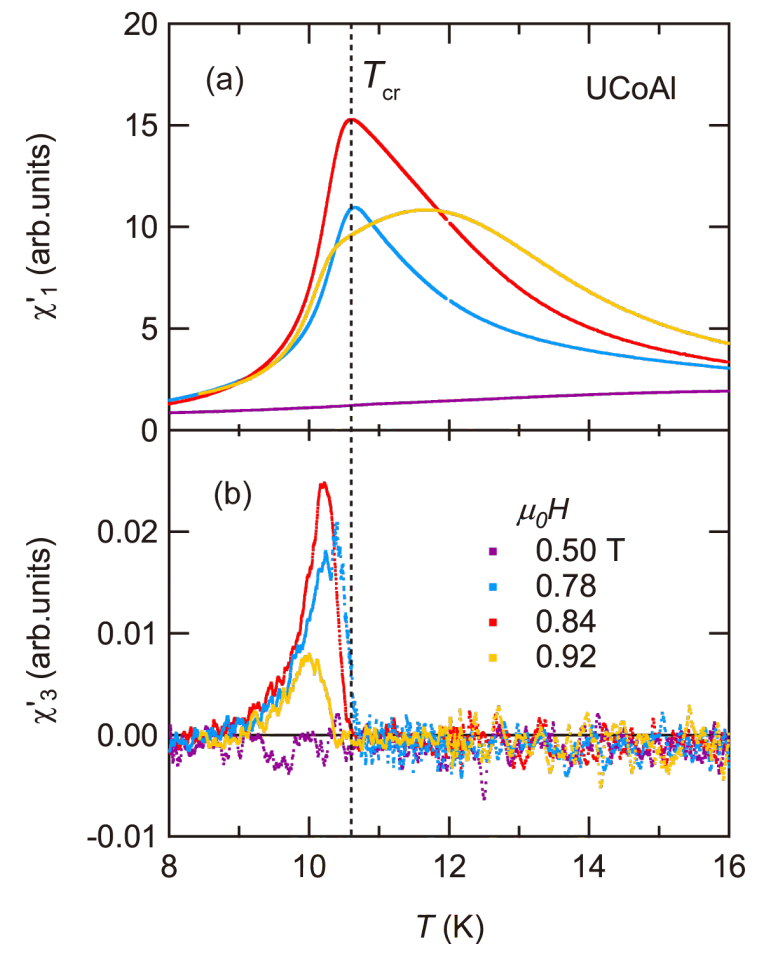

Figure 1. Temperature dependence of (a) $\chi_{1}^{\prime}$ and (b) $\chi_{3}^{\prime}$ at several magnetic fields. The dashed line is drawn at $10.6 \mathrm{~K}$ $\left(=T_{\mathrm{cr}}\right)$.

Figure 1 (a) shows the temperature dependence of $\chi_{1}^{\prime}$ at several magnetic fields. The behavior of $\chi_{1}^{\prime}$ qualitatively reproduced the previous report [7]. $\chi_{1}^{\prime}$ develops in the vicinity of the CP (see also Figure 4 (a)). Since $\chi_{1}$, proportional to $\chi_{1}^{\prime}$ in the paramagnetic region, should diverge toward the $\mathrm{CP}$, we determined the $\mathrm{CP}$ from the peak position of $\chi_{1}^{\prime}$. The location of the CP was determined to be $\left(T_{\mathrm{cr}}, H_{\mathrm{cr}}\right)=(10.6 \pm 0.2 \mathrm{~K}$, $0.84 \pm 0.04 \mathrm{~T}$ ).

Figure 1 (b) shows the temperature dependence of $\chi_{3}^{\prime} . \chi_{3}^{\prime}(T)$ shows a rather sharp peak compared to $\chi_{1}^{\prime}$ and is approximately zero just above $T_{\mathrm{cr}}$. The peak height in $\chi_{3}^{\prime}(T)$ is maximum at $H=H_{\mathrm{cr}}$.

Here, we mention the experimental conditions of the measurement of $\chi_{3}^{\prime}$. It is known for ferromagnets that $\chi_{3}^{\prime}$ is sensitive to the excitation field amplitude $h_{0}$ [12]. A large amplitude will increase the higher-order terms that we ignored at Eq. (5). A high frequency will also affect $\chi_{3}^{\prime}$ by inducing eddy current that shifts the phase of the excitation field and thus attenuates its amplitude inside the sample [11]. These effects might distort the shape in $\chi_{3}^{\prime}(T)$ and lead to misunderstanding of its behavior. Ideally, the frequency and amplitude should be as low and small as possible. Figure 2 shows the amplitude and frequency dependences of $\chi_{3}^{\prime}(T)$. As seen in Figure 2 (a), the behavior of $\chi_{3}^{\prime}(T)$ depends on the amplitude $h_{0}$. A small dip seen just below $T_{\text {cr }}$ grows with increasing the amplitude. The dip is not observed at 0.98 Oe within the experimental accuracy. 


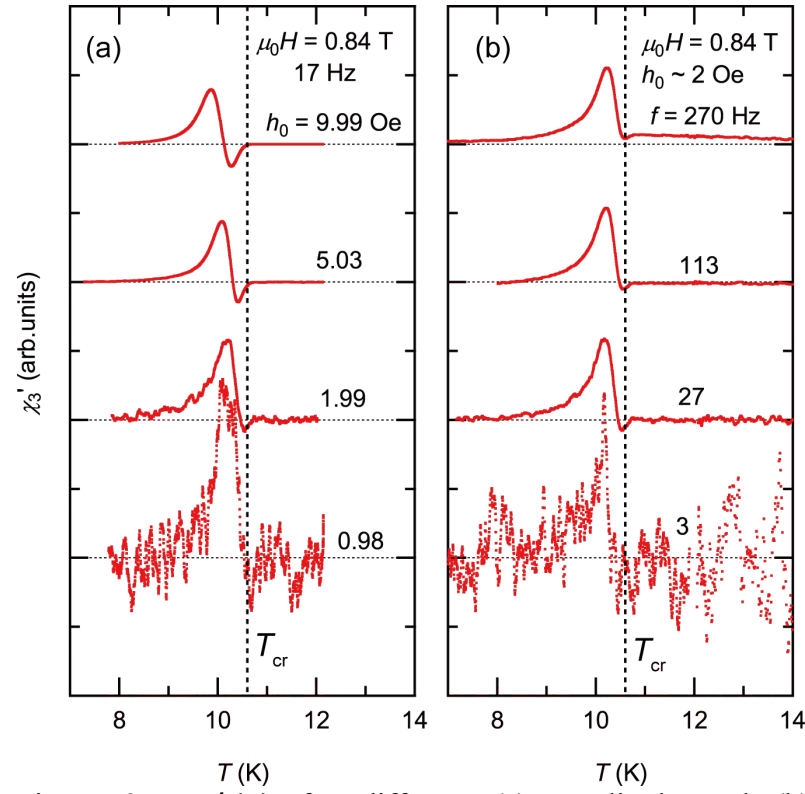

Figure 2. $\chi_{3}^{\prime}(T)$ for different (a) amplitude and (b) frequency of the excitation field. The dashed lines are drawn at $10.6 \mathrm{~K}\left(=T_{\mathrm{cr}}\right)$

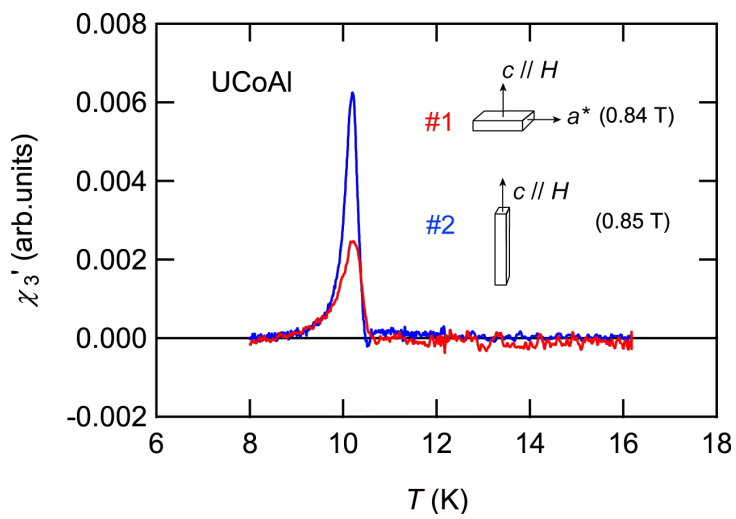

Figure 3. $\chi_{3}^{\prime}$ measured in different samples. Sample \#2 has a smaller demagnetization factor than that of sample \#1. Note that these two samples were measured in different pick-up coils. Therefore, comparing the amplitude of each data is meaningless.

As seen in Figure 2 (b), the frequency dependence of $\chi_{3}^{\prime}(T)$ is rather weak. The behaviors for $27 \mathrm{~Hz}$ and 113 $\mathrm{Hz}$ are almost the same. $\chi_{3}^{\prime}(T)$ for $3 \mathrm{~Hz}$ displays a poor signal-to-noise ratio but its behavior is roughly identical to that for $27 \mathrm{~Hz} . \chi_{3}^{\prime}(T)$ for $270 \mathrm{~Hz}$ has a similar behavior to those for the lower frequencies although $\chi_{3}^{\prime}$ above $T_{\text {cr }}$ is finite. We conclude therefore that the influence of the frequency emerges above $113 \mathrm{~Hz}$ and a lower frequency provides an inherent behavior of $\chi_{3}$. The above results guarantee that our selected condition, $f$ $=67 \mathrm{~Hz}$ and $h_{0}=0.98 \mathrm{Oe}$, is sufficient to measure $\chi_{3}$ properly.

We also checked the demagnetization effect, which is known to affect the higher-order susceptibility stronger than the linear susceptibility [13]. In Figure 3, we show $\chi_{3}^{\prime}(T)$ of another sample (sample \#2) together with that of sample \#1. Since sample \#2 is long along the $c$-axis,
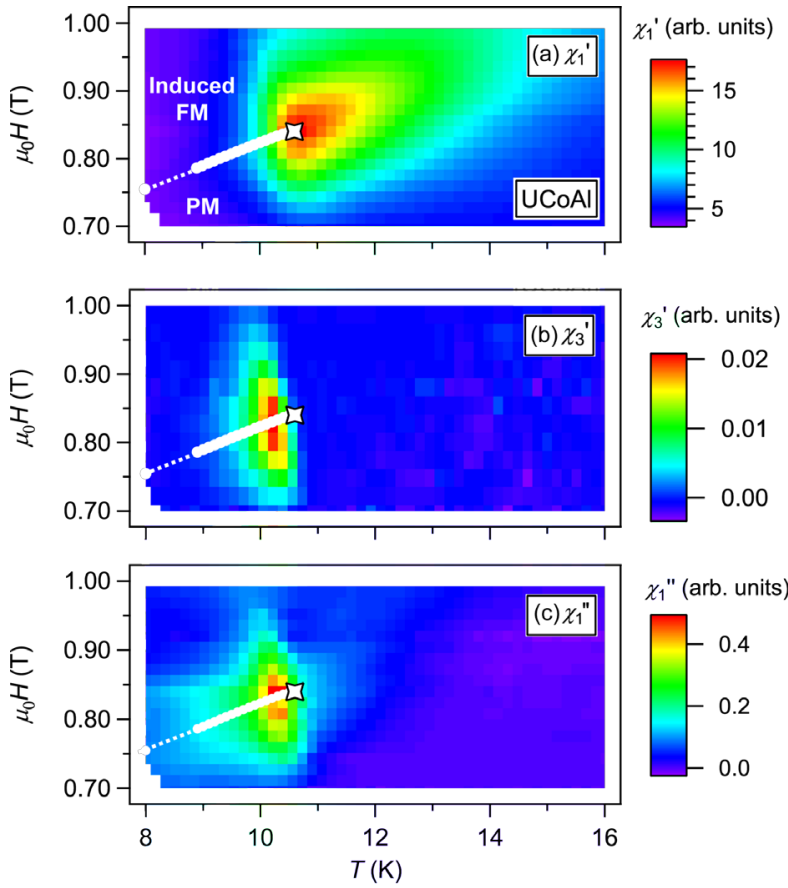

Figure 4. Color maps of (a) $\chi_{1}^{\prime}$, (b) $\chi_{3}^{\prime}$, and (c) $\chi_{1}^{\prime \prime}$, which are constructed from the data taken at $\mu_{0} H=0.70,0.75,0.78$, $0.81,0.84,0.86,0.88,0.90,0.92,0.95$ and $1.00 \mathrm{~T}$. The white circles indicate the metamagnetic transition points, determined from the inflection point of the magnetization curve. The dashed lines are the guides for the eyes. The white stars represent the $\mathrm{CP}$.

the demagnetization factor is smaller than that of sample $\# 1$. The obtained $\chi_{3}^{\prime}(T)$ was qualitatively the same as that of sample \#1, indicating that the demagnetization effect is not serious to discuss the qualitative behavior of $\chi_{3}^{\prime}(T)$ in our measurement. Consequently, we confirmed that the observed behavior of $\chi_{3}^{\prime}(T)$ is intrinsic. The metamagnetic $\mathrm{CP}$ of UCoAl can be determined as the onset of the maximum peak of $\chi_{3}^{\prime}$.

\section{2. $\chi_{3}^{\prime}$ at the critical point}

The significant feature of $\chi_{3}^{\prime}(T)$ in UCoAl is that $\chi_{3}^{\prime}=0$ for $T>T_{\mathrm{cr}}$. This result means that critical divergence is absent or emerges in a very narrow range in temperature. One of the possible reasons is that the critical divergence emerges from a temperature very close to $T_{\mathrm{cr}}$. Indeed, the temperature range of the criticality in $\mathrm{Ni}\left(T_{\mathrm{C}}=630 \mathrm{~K}\right)$ is narrower than $1 \mathrm{~K}[11]$. $T_{\mathrm{cr}}$ of $\mathrm{UCoAl}$ is more than sixty times smaller than that of Ni. The other possibility is that $\chi_{3}$ of UCoAl is inherently small in amplitude or absent. $\chi_{3} \propto$ $d^{3} M / d H^{3}$ will be very small if the magnetization curve is almost linear. To understand the microscopic nature of $\chi_{3}^{\prime}$, further detailed study are required.

The feature of $\chi_{3}^{\prime}$ is clearly seen when we compare the color maps of $\chi_{1}^{\prime}, \chi_{3}^{\prime}$, and $\chi_{1}^{\prime \prime}$ in the $H-T$ phase diagram, as shown in Figure 4. A finite $\chi_{3}^{\prime}$ is observed in a relatively limited area compared to $\chi_{1}^{\prime}$. The behavior of $\chi_{3}^{\prime}$ is similar to the imaginary part of the linear susceptibility, $\chi_{1}^{\prime \prime}$, which reflects the energy loss due to magnetic hysteresis. However, the onset of the 
maximum peak of $\chi_{3}^{\prime}$, corresponding to the $\mathrm{CP}$, is much clearer than that of $\chi_{1}^{\prime \prime}$. One may consider that $\chi_{3}$ emerges in the hysteresis region. However, because magnetic dynamics is complicated in the hysteresis region, it is difficult to discuss $\chi_{3}$ in this region. We would like to emphasis that the CP can be determined as the onset of $\chi_{3}^{\prime}$, which is an explicit definition and can be a good probe for determining the $\mathrm{CP}$, at least, in this system.

\section{Conclusion}

In conclusion, we have measured the third harmonics of the AC magnetic susceptibility of UCoAl. This is, to our knowledge, the first report of $\chi_{3}^{\prime}$ in the itinerant-electron metamagnetic system. $\chi_{3}^{\prime}$ has a single peak structure near the $\mathrm{CP}$ in the hysteresis region. The metamagnetic CP corresponds to the onset of the maximum peak in $\chi_{3}^{\prime}$. Therefore, the measurement of $\chi_{3}^{\prime}$ can be a new useful tool to determine the CP. We also expect that it will be helpful to determine the QCP more precisely.

\section{Acknowledgements}

The author would like to thank N. Kabeya and A. Ochiai for helpful discussions. We also thank $\mathrm{M}$. Kikuchi, H. Moriyama, and N. Fukiage for their technical support. This work was supported by JPSJ KAKENHI Grant Numbers 23654109 and 26400345. This work was also performed under the Inter-University Cooperative Research Program of the Institute for Materials Research, Tohoku University (Proposal Numbers 16K0208 and 17K0201).

\section{References}

[1] N.D. Mathur, F.M. Grosche, S.R. Julian, I.R. Walker, D.M. Freye, R.K.W. Haselwimmer and G.G. Lonzarich, Magnetically mediated superconductivity in heavy fermion compounds, Nature 394 (1998), pp. 39-43.

[2] R.A. Borzi, S.A. Grigera, J. Farrell, R.S. Perry, S.J. S. Lister, S.L. Lee, D.A. Tennant, Y. Maeno and A.P. Mackenzie, Formation of a nematic fluid at high fields in $\mathrm{Sr}_{3} \mathrm{Ru}_{2} \mathrm{O}_{7}$, Science 315 (2007), pp. 214-217.
[3] S.S. Saxena, P. Agarwal, K. Ahilan, F.M. Grosche, R.K.W. Haselwimmer, M.J. Steiner, E. Pugh, I.R. Walker, S.R. Julian, P. Monthoux, G.G. Lonzarich, A. Huxley, I. Sheikin, D. Braithwaite and J. Flouquet, Superconductivity on the border of itinerant-electron ferromagnetism in UGe2, Nature 406 (2000), pp. 587-592.

[4] F. Levy, I. Sheikin, B. Grenier and A.D. Huxley, Magnetic field-induced superconductivity in the ferromagnet URhGe, Science 309 (2005), pp. 1343-1346.

[5] P. Javorsky, V. Sechovsky, J. Schwenzer, F. Bourdarot, E. Lelievre-Berna, A.V. Andreev and Y. Shiokawa, Magnetization densities in UCoAl studied by polarized neutron diffraction, Phys. Rev. B 63 (2001), 064423.

[6] D. Aoki, T. Combier, V. Taufour, T.D. Matsuda, G. Knebel, H. Kotegawa and J. Flouquet, Ferromagnetic quantum critical endpoint in UCoAl, J. Phys. Soc. Jpn. 80 (2011), p.094711.

[7] N. Kimura, N. Kabeya, H. Aoki, K. Ohyama, M. Maeda, H. Fujii, M. Kogure, T. Asai, T. Komatsubara, T. Yamamura and I. Satoh, Quantum critical point and unusual phase diagram in the itinerant-electron metamagnet UCoAl, Phys. Rev. B 92 (2015), p.035106.

[8] S. Fujiki and S. Katsura, Nonlinear susceptibility in the spin-glass, Prog. Theor. Phys. 65 (1981), pp. 1130-1144.

[9] Y. Bitla and S.N. Kaul, Mean-field treatment of nonlineat susceptibilities for a ferromagnet of arbitrary spin, EPL 96 (2011), p.37012.

[10] T. Sato and Y. Miyako, Nonlinear susceptibility and specific-heat of $\left(\mathrm{Pd}_{0.9966} \mathrm{Fe}_{0.0034}\right)_{0.95} \mathrm{Mn}_{0.05}, J$. Phys. Soc. Jpn. 51 (1982), pp. 1394-1400.

[11] T. Shirane, T. Moriya, T. Bitoh, A. Sawada, H. Aida and S. Chikazawa, Nonlinear susceptibility of Ni near the Curie-temperature, J. Phys. Soc. Jpn. 64 (1995), pp. 951-959.

[12] T. Bitoh, T. Shirane and S. Chikazawa, Critical-behavior of linear and nonlinear susceptibilities near Curie-temperature in $\mathrm{Au}_{82} \mathrm{Fe}_{18}$ alloy, J. Phys. Soc. Jpn. 62 (1993), pp. 2837-2844.

[13] B. Ozcelik, K. Kiymac, J.C. Verstelle, A.J. Vanduyneveldt and J.A. Mydosh, Linear and nonlinear Ac susceptibilities of the spin-glass $\mathrm{Eu}_{0.4} \mathrm{Sr}_{0.6} \mathrm{~S}$, J. Phys.: Condens. Matter 4 (1992), pp. 6639-6650. 